\title{
Oral cancer cells sustainedly infected with Porphyromonas gingivalis exhibit resistance to Taxol and have higher metastatic potential
}

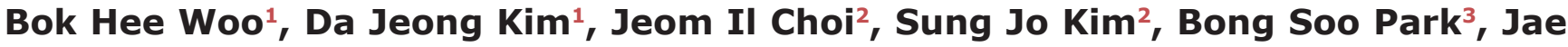 \\ Min Song ${ }^{4}$, Ji Hye Lee ${ }^{1,5}$ and Hae Ryoun Park ${ }^{1,5}$ \\ ${ }^{1}$ Department of Oral Pathology \& BK21 PLUS Project, School of Dentistry, Pusan National University, Mulgeum-up, Yangsan \\ 50612, South Korea \\ ${ }^{2}$ Department of Periodontology, School of Dentistry, Pusan National University, Mulgeum-up, Yangsan 50612, South Korea \\ ${ }^{3}$ Department of Oral Anatomy, School of Dentistry, Pusan National University, Mulgeum-up, Yangsan 50612, South Korea \\ ${ }^{4}$ Department of Oral and Maxillofacial Surgery, School of Dentistry, Pusan National University, Mulgeum-up, Yangsan 50612, \\ South Korea \\ ${ }^{5}$ Institute of Translational Dental Sciences, Pusan National University, Mulgeum-up, Yangsan 50612, South Korea \\ Correspondence to: Hae Ryoun Park, email: parkhr@pusan.ac.kr \\ Keywords: Porphyromonas gingivalis, oral cancer, Taxol, Notch 1, metastasis
}

Abbreviations: oral squamous cell carcinoma, OSCC; cancer stem cell, CSC; Notch intracellular domain, NICD

Received: January 25, $2017 \quad$ Accepted: March 03, $2017 \quad$ Published: March 24, 2017

Copyright: Woo et al. This is an open-access article distributed under the terms of the Creative Commons Attribution License 3.0 (CC BY 3.0), which permits unrestricted use, distribution, and reproduction in any medium, provided the original author and source are credited.

\section{ABSTRACT}

Major obstacles to improving the prognosis of patients with oral squamous cell carcinoma (OSCC) are the acquisition of resistance to chemotherapeutic agents and development of metastases. Recently, inflammatory signals are suggested to be one of the most important factors in modulating chemoresistance and establishing metastatic lesions. In addition, epidemiological studies have demonstrated that periodontitis, the most common chronic inflammatory condition of the oral cavity, is closely associated with oral cancer. However, a correlation between chronic periodontitis and chemoresistance/ metastasis has not been well established. Herein, we will present our study on whether sustained infection with Porphyromonas gingivalis, a major pathogen of chronic periodontitis, could modify the response of OSCC cells to chemotherapeutic agents and their metastatic capability in vivo. Tumor xenografts composed of $P$. gingivalis-infected OSCC cells demonstrated a higher resistance to Taxol through Notch1 activation, as compared with uninfected cells. Furthermore, P. gingivalis-infected OSCC cells formed more metastatic foci in the lung than uninfected cells.

\section{INTRODUCTION}

Oral squamous cell carcinoma (OSCC), which is the most common cancer of the oral cavity, is mainly treated with surgery alone. Because surgical treatment of OSCC sacrifices both important functionality and esthetics of the head and neck, surgical treatment of OSCC should be minimized or replaced with other treatment modalities such as chemotherapy and radiotherapy. However, chemotherapy is used as an adjuvant therapy, not as a single treatment modality, for OSCC because it rarely results in a cure and has demonstrated limited effectiveness in OSCC [1]. Therefore, improvement of OSCC responsiveness to chemotherapeutic agents is essential for patients with OSCC to obtain a better functional and cosmetic result from treatment that could be obtained through a deeper understanding of the reasons or mechanism involved in the low sensitivity of OSCC cells to chemotherapeutic agents.

Recently, the importance of signals from the tumor microenvironment that is composed of extracellular matrix, fibroblasts, and immune cells has been emphasized. Among the numerous factors in the microenvironment, signals of inflammatory stimuli confer resistance to chemotherapy and 
tumor progression $[2,3]$. Accordingly, associations between inflammation and cancer cells have been extensively studied in a variety of cancers such as gastric, lung, and colorectal cancer, suggesting that inflammatory mediators are linked to therapeutic resistance and increased aggressiveness of tumor cells, including their invasive and metastatic abilities $[3,4]$. It has been suggested that inflammation contributes to the formation, maintenance, and expansion of pluripotent cancer stem cells (CSCs), a small subpopulation of tumor cells with the capability of self-renewal, dedifferentiation, and tumorigenicity $[5,6]$. Notably, CSCs are also thought to be a major reason why cancer cells demonstrate aggressive behaviors such as chemoresistance and metastasis. This hypothesis can be supported by numerous studies including observations that CSCs are expanded and that prostaglandin $\mathrm{E} 2$, an inflammatory mediator, induces CSC expansion and accelerates the formation of liver metastases in mice by activating $\mathrm{NF \kappa B}[7,8]$. Therefore, it can be hypothesized that inflammatory signals from the microenvironment modulate the responsiveness of cancer cells to chemotherapeutic agents through the acquisition of stem cell properties.

Chronic periodontitis is one of the most common chronic inflammatory diseases of the oral cavity, especially in older patients. A relationship between periodontal diseases and oral cancer is strongly suspected because of the common occurrence of periodontitis, but this correlation has been investigated only in several epidemiological studies and a few experimental studies. The epidemiological studies demonstrated a higher risk of oral cancer in patients with periodontal disease [9-12], and experimental studies indicated that Porphyromonas gingivalis, a major pathogen involved in chronic periodontitis, increased the invasive capability of OSCC cells through the production of MMP-2 and 9 [13]. Recently, we also observed that acute and chronic exposure to $P$. gingivalis increased the migratory and invasive properties of oral cancer cells, with a higher secretion of MMPs [14]. In addition, it has been shown that prolonged and repeated chronic exposure to $P$. gingivalis helped provide OSCC cells with resistance to Taxol by promoting CSC properties, suggesting that chronic periodontitis plays a role in the chemoresistance of OSCC cells [15]. Although studies on a variety of cancers have been conducted for the association between cancer stemness and resistance to chemotherapy, there are few reports on a direct link between inflammation and chemoresistance. Furthermore, to our knowledge, no in vivo study has been performed on chronic periodontitis and the responsiveness of oral cancer to chemotherapeutic reagents. To clarify whether chronic periodontitis could modify the susceptibility of OSCC to chemotherapeutic agents in vivo, we examined whether prolonged and repeated $P$. gingivalis infection mimicking chronic periodontitis could affect the responsiveness of tumor xenografts to Taxol in mice. In addition, we investigated the mechanism involved in the chemoresistance of OSCC cells that were sustainedly infected with $P$. gingivalis, especially on the Notch pathway, because the most recent in vitro studies consistently suggested that Notch signaling promotes several malignant features of migration, invasion [16], chemoresistance [17], and stemness [18].

\section{RESULTS}

\section{Slower in vivo tumor growth was exhibited by OSCC cells sustainedly infected with $P$. gingivalis than noninfected OSCC cells}

It has been shown that Ca9-22 and SCC25 OSCC cells infected with $P$. gingivalis showed lower proliferative activity than non-infected cells [19]. To further confirm that $P$. gingivalis infection contributes to the slower growth of OSCC cells, we infected OSC-20 OSCC cells with $P$. gingivalis twice and observed growth potential of $P$. gingivalis-infected and non-infected cells. As expected, $P$. gingivalis-infected OSC-20 cells displayed retarded growth in comparison with non-infected cells (Figure 1A). To investigate whether chronic periodontitis could affect the growth of oral cancer in vivo, we compared the tumor volume induced by sustainedly $P$. gingivalis-infected OSCC cells with that of uninfected control OSCC cells in nude mice. Briefly, cells were repeatedly infected with $P$. gingivalis twice a week for 5 weeks. Then the right flank of a mouse was injected with the infected OSC-20 cells, and the left flank of the same mouse was injected with uninfected OSC-20 cells. To rule out potential animal-to-animal variations, infected and uninfected tumor cells were simultaneously implanted in the same mouse (Figure 1B). Tumor volume was measured once a week, starting 8 days $(1 \mathrm{w})$ after subcutaneous implantation of tumor cells. As we continued to monitor tumor growth during the following weeks, we were able to detect marked increases in tumor volume on both sides; tumors induced by uninfected control OSC-20 cells were significantly larger than those induced by $P$. gingivalis-infected cells (Figure 1C). The slower tumor growth observed with $P$. gingivalis infected cells indicated that chronic infection with $P$. gingivalis could slow tumor growth in an OSCC xenograft mouse model. At Thirty-five days after inoculation, the tumor masses were excised, sectioned, and stained with $\mathrm{H}$ \& $\mathrm{E}$. These sections displayed histopathologic findings of OSCC, with prominent central necrosis (Figure 1D, left panels). At high magnification, the OSCC cells showed marked pleomorphism, little keratin production, and high mitotic activity, which are characteristics of moderately differentiated OSCC (Figure 1D, right panels). There was no definitive difference in histologic grade or morphological features between tumors induced by uninfected and $P$. gingivalisinfected OSC-20 cells.

\section{Sustained infection with $P$. gingivalis activated Notch1 in OSCC cells}

Our previous report demonstrated that prolonged and repeated infection of Ca9-22 OSCC cells with $P$. gingivalis 
induced CSC properties [15]. The Notch signaling pathway is known to play a critical role in maintaining the CSC population [20]. Thus, we investigated the expression of cleaved Notch1 (Notch intracellular domain, NICD), its active form, in OSC-20 cells chronically infected with $P$. gingivalis. As expected, protein levels of NICD were significantly increased over time in OSC-20 cells with a $P$. gingivalis infection (Figure 2A). In addition, increased levels of NICD in $P$. gingivalis-infected cells were observed in other types of OSCC cells lines (Supplementary Figure 1). Such activation of Notch1 in infected OSC-20 cells was further confirmed with significant stimulation of CSL promoter activity detected by a luciferase assay (Figure 2B), as well as changes in the mRNA levels of Hesl and Heyl, putative downstream targets of active Notch1 (Figure 2C, middle and right panels). With the presence of $P$. gingivalis within the OSCC cells that was verified by the expression of $16 \mathrm{~S}$ rRNA in cell lysates (Figure 2C, left panel), these data indicate that $P$. gingivalis induced activation of Notch1 in OSCC cells.

\section{Sustained infection of OSCC cells with $P$. gingivalis caused resistance to Taxol through Notch1 activation}

To determine the role of activated Notch1 with chemotherapy in oral cancers, the viability of NICDoverexpressing OSC-20 cells was assessed in the presence of Taxol, a well-characterized chemotherapeutic agent. When we first examined cellular toxicity to Taxol in naïve OSC-20 cells, their viability was gradually decreased in a dose-dependent manner at both 24 and 48 $\mathrm{h}$ of Taxol incubation (Figure 3A). Importantly, NICDoverexpressing OSC-20 cells exhibited higher viability than control cells transfected with an empty vector in the presence of Taxol. Such resistance to Taxol toxicity was prominent $24 \mathrm{~h}$ following a drug treatment, but not at a later time point (48 h) (Figure 3B). This is an interesting parallel to our previous finding that indicated reduced susceptibility to Taxol in Ca9-22 cells, another OSCC cell, upon chronic exposure to $P$. gingivalis. Moreover, these

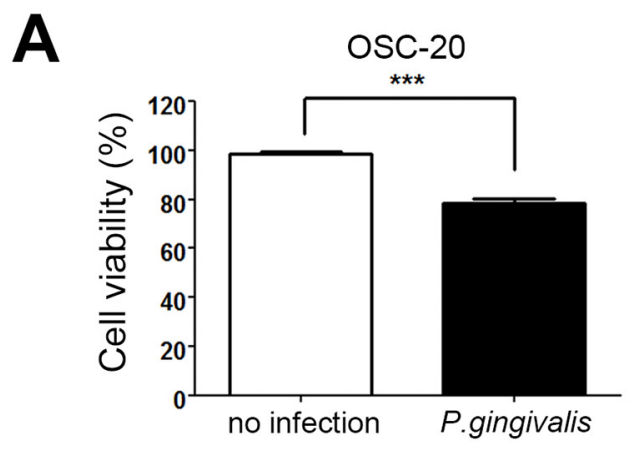

B
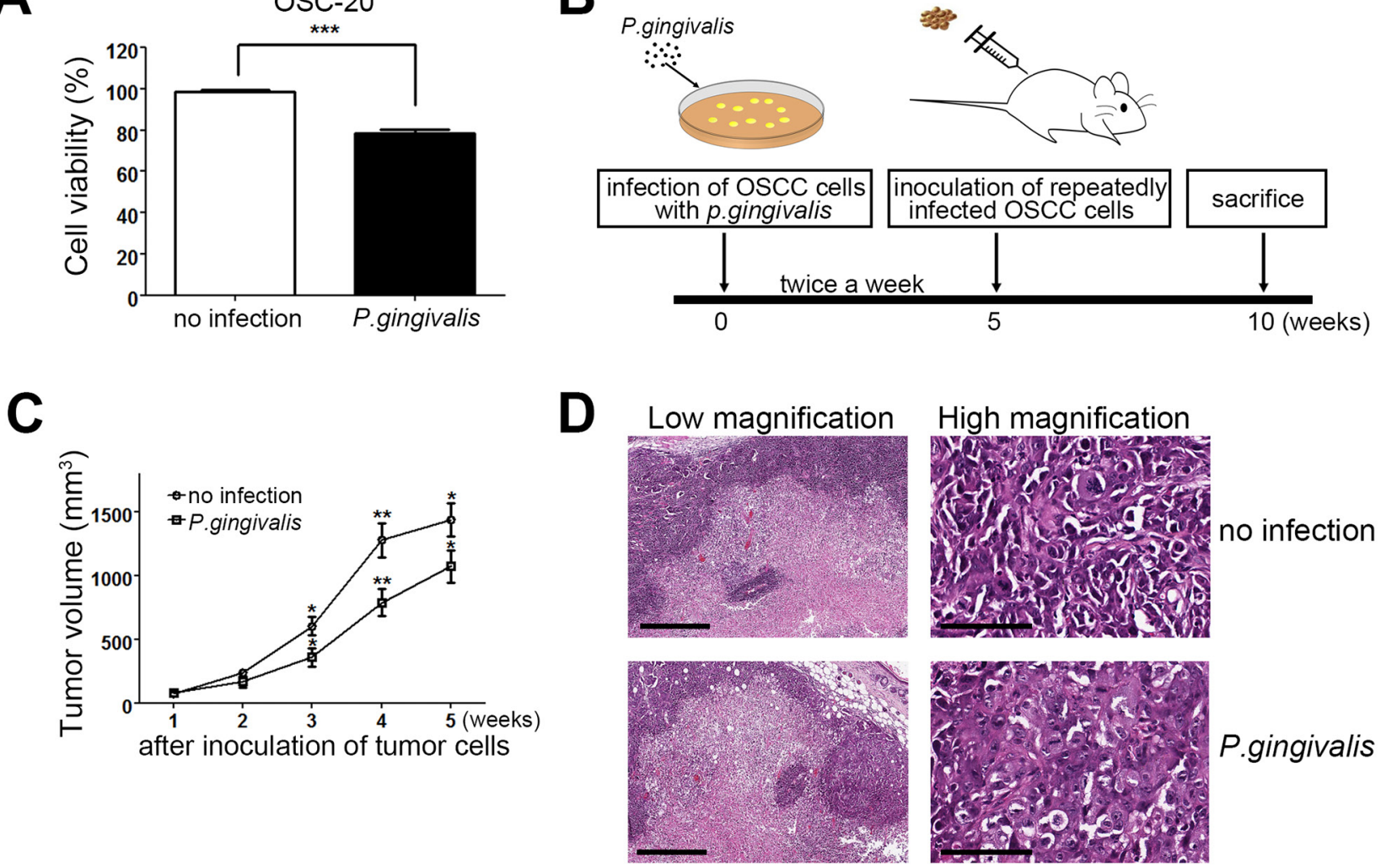

Figure 1. Tumor mass growth was slowed in OSCC cells with a sustained $\boldsymbol{P}$. gingivalis infection. (A) OSC-20 OSCC cells were infected with $P$. gingivalis at a MOI of 100 for $2 \mathrm{~h}$ and cultured for an additional $24 \mathrm{~h}$. Relative growth rates of $P$. gingivalis-infected cells to non-infected cells were calculated based on a MTT assay. (B) To study the effect of $P$. gingivalis on tumor growth, non-infected and chronically infected OSC-20 cells with $P$. gingivalis were injected into the right and left flanks, respectively, of nude mice. (C) Tumor volume was measured once a week after subcutaneous implantation of tumor cells. Data are presented as mean \pm standard deviation. Significance was assessed using a paired Student's $t$ test. ${ }^{*}, P<0.05$; ** $P<0.01$. (D) Tumor masses involving $P$. gingivalis-infected or uninfected OSC-20 cells were excised, sectioned, and stained with H \& (E) Representative images of H \& E stained sections were obtained. Scale bars, $500 \mu \mathrm{m}$ (left panels), $100 \mu \mathrm{m}$ (right panels). 
findings led to the hypothesis that $P$. gingivalis contributes to and induces activation of Notch 1 that leads to the development of Taxol resistance in various OSCC cells.

To validate the idea that $P$. gingivalis induces Taxol resistance in vivo, a mouse xenograft model was established using OSC-20 cells. OSC-20 cells with a chronic $P$. gingivalis infection and uninfected OSC-20 cells were implanted subcutaneously into the right and left flanks, respectively, of nude mice. Application of Taxol $(10 \mathrm{mg} / \mathrm{kg})$ or vehicle via intraperitoneal injections was initiated 14 days after tumor inoculation and was continued twice a week for a 4-week period. When OSC-20-induced tumors were compared between $P$. gingivalis-infected and control xenografts following repeated Taxol treatment, we found a greater increase in size associated with $P$. gingivalis infection, albeit smaller initial measurements before application of Taxol $(\mathrm{N}=6, P<0.05$; Figure $3 \mathrm{C}$, left panel). In addition, the suppressive effect of Taxol on the growth rate of tumors was significantly greater in control xenografts, as compared with that of $P$. gingivalisinfected OSC-20 cells (Figure 3C, right panel), indicating lower sensitivity to Taxol in $P$. gingivalis-infected tumor xenografts. To examine whether an association could be established in vivo between the $P$. gingivalis-induced activation of Notch1 and chemoresistance demonstrated in vitro (Figure 2), we monitored the NICD levels in tumor xenografts with $P$. gingivalis-infected and control OSC20 cells. Importantly, the level of NICD was significantly higher in $P$. gingivalis-infected tumor xenografts compared with uninfected controls (5 out of 6 pairs, Figure 3D), suggesting that the $P$. gingivalis-induced activation of Notch1 was responsible for the development of chemoresistance in vivo.

NICD-associated resistance to Taxol in $P$. gingivalis-infected tumors was further confirmed by coapplication of DAPT, an inhibitor of Notch activation, as well as Taxol in a xenograft model (Figure 4A). In the presence of DAPT, the effect of Taxol as a tumor growth suppressor was clearly visible in both $P$. gingivalisinfected and uninfected xenografts (Figure 4B, left panel). However, as Taxol was repeatedly applied, a more prominent retardation of tumor growth was observed in $P$. gingivalis-infected xenografts (Figure 4B, right panel, $P<0.05)$. Enhanced sensitivity to Taxol following
A

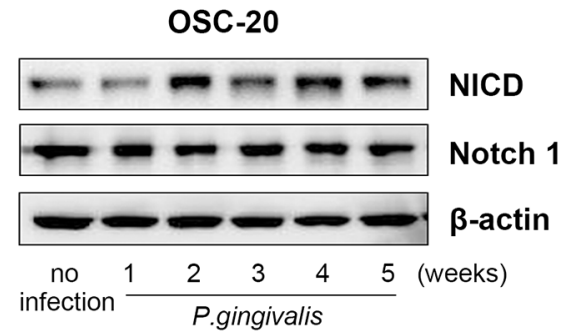

C

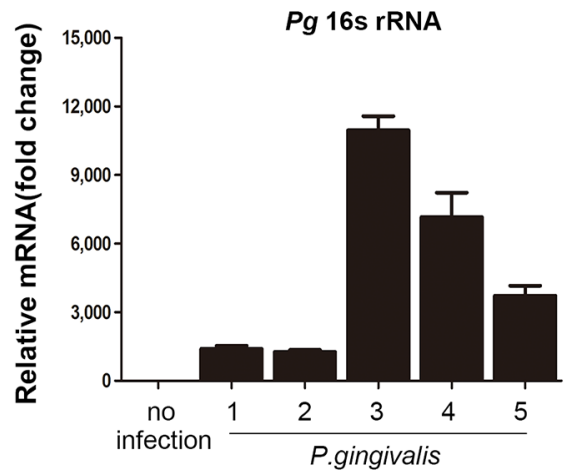

B

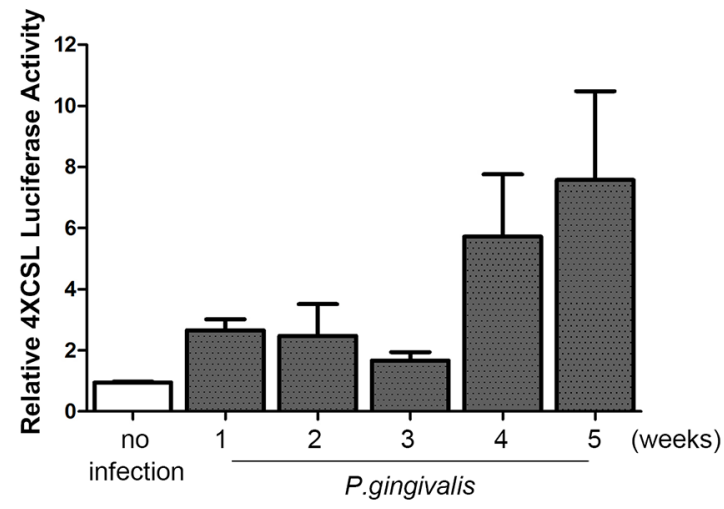

Hey 1

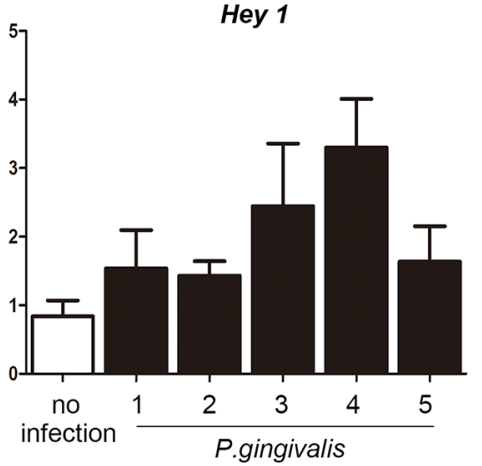

Figure 2. Notch1 was activated in OSCC cells with a sustained $\boldsymbol{P}$. gingivalis infection. (A) To mimic chronic infection with $P$. gingivalis, OSC-20 cells were repeatedly infected with $P$. gingivalis twice a week for the indicated periods. Notch1 and Notch intracellular domain (NICD) were analyzed using a Western blot analysis. (B) Cells were cotransfected with $4 \times$ CSL promoter-luciferase construct and a constitutively active Renilla luciferase plasmid. The graphs demonstrate relative luciferase activity. (C) The presence of $P$. gingivalis was confirmed by identifying $16 \mathrm{~S}$ rRNA using real-time PCR. In addition, the levels of Heyl and Hes 1 , as well as downstream Notch targets, were investigated. 
A

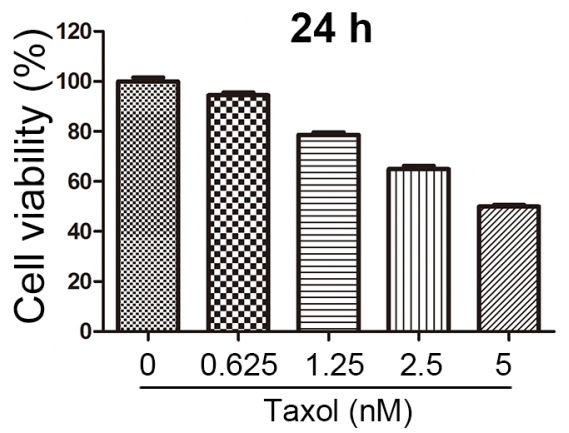

B

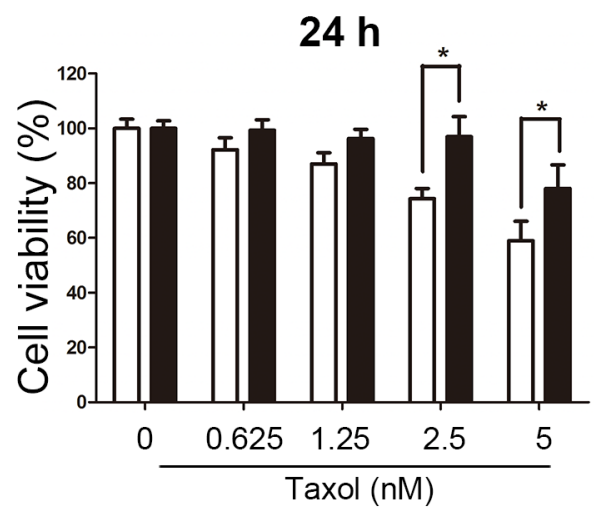

C

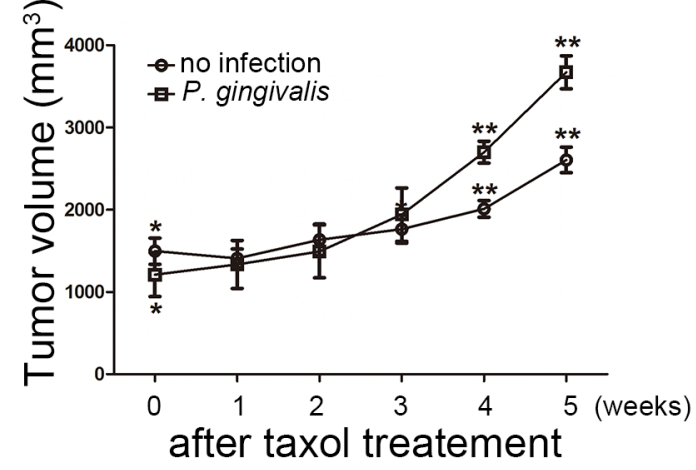

D
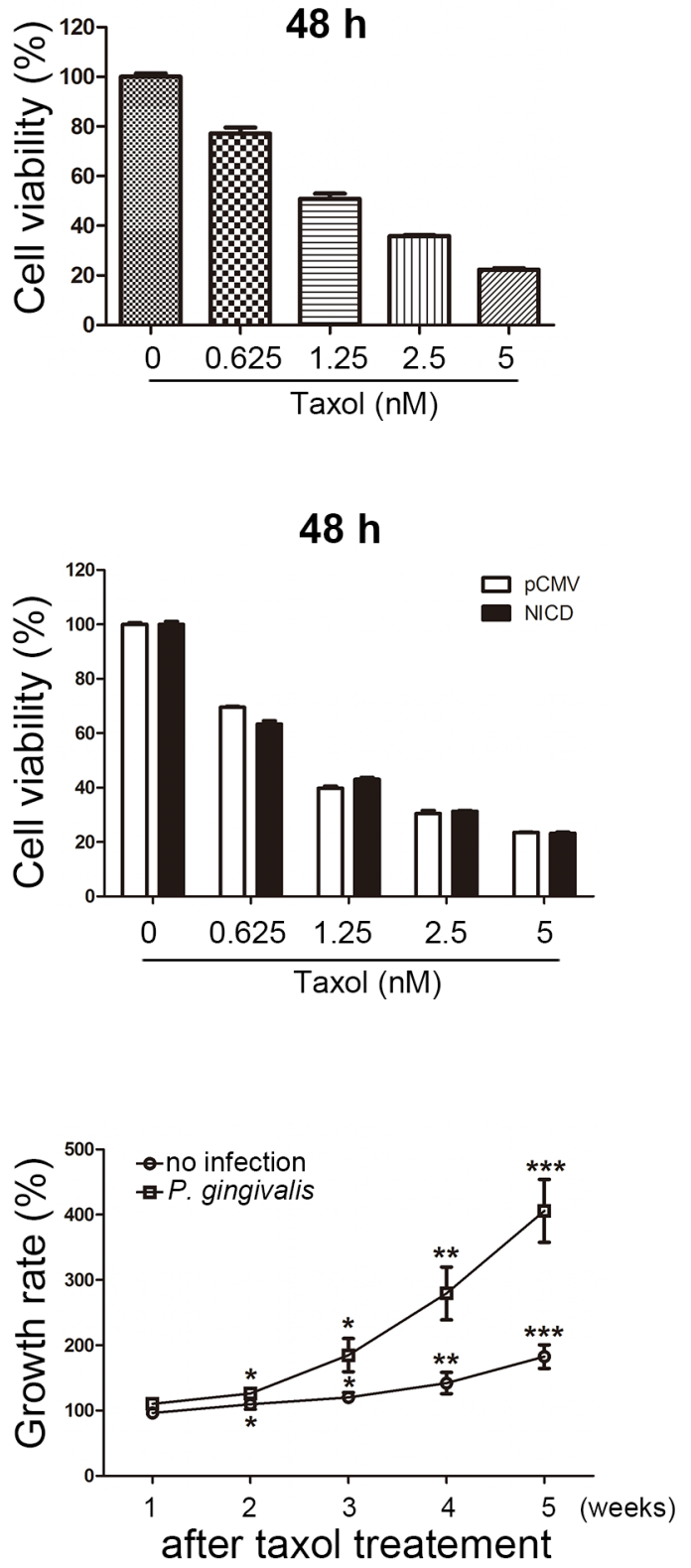

$48 \mathrm{~h}$

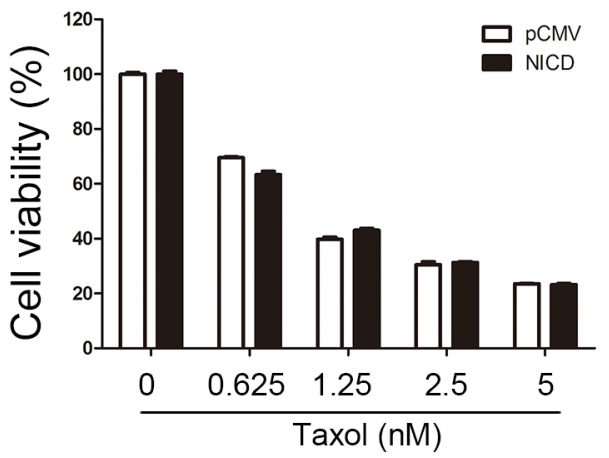

\section{NICD}

Notch 1

$\beta$-actin

Figure 3. Overexpression of NICD decreases Taxol-induced cell death in OSCC cells. (A) Cells were treated with various concentrations of Taxol $(0,0.625,1.25,2.5$, and $5 \mathrm{nM})$ for 24 or $48 \mathrm{~h}$ and then subjected to an MTT assay. (B) Cell viability was analyzed in Taxol-exposed cells pretreated with or without NICD transfection. (C) OSC-20 cells with a sustained $P$. gingivalis infection and uninfected OSC-20 cells were implanted subcutaneously into the right and left flanks, respectively, of nude mice. Applications of Taxol (10 mg/kg) or vehicle through intraperitoneal injections were initiated 14 days after tumor inoculation and continued twice a week for a 4-week period. Data are presented as mean \pm standard deviation. Significance was assessed using a paired Student's $t$ test. *, $P<0.05$; ${ }^{* *}, P<0.01$; ***, $P<0.001$. (D) NICD expression was analyzed in tumor xenografts of $P$. gingivalis-infected OSCC cells using Western blot. 
inhibition of $P$. gingivalis-induced Notch signaling was demonstrated to play a central role in the development of chemoresistance with chronic exposure of OSCC cells to P. gingivalis.

\section{Sustained infection with $P$. gingivalis increased the metastatic potential of OSCC cells}

For cancer cells to establish a metastatic focus as a tumor mass, they travel a long distance within the blood stream. Therefore, cancer cells should survive in a movable and unattached state before their resettlement at metastatic sites such as the lungs, liver, or bones.
We first examined the viability of OSC-20 OSCC cells with or without chronic exposure to $P$. gingivalis in a nonadherent and continuously agitated setting. The viability of both cell types decreased in a time-dependent manner, but $P$. gingivalis-infected OSC-20 cells were more resilient to such mechanical agitation, as compared with uninfected controls (Figure 5A, $P<0.05$ ), suggesting that $P$. gingivalis-infected OSCC cells could withstand mechanical stress and survive within the blood stream, thus capable of forming metastatic foci.

To validate the idea that chronic $P$. gingivalis infection could promote metastasis through the blood stream in vivo, the metastatic abilities of uninfected and $P$.
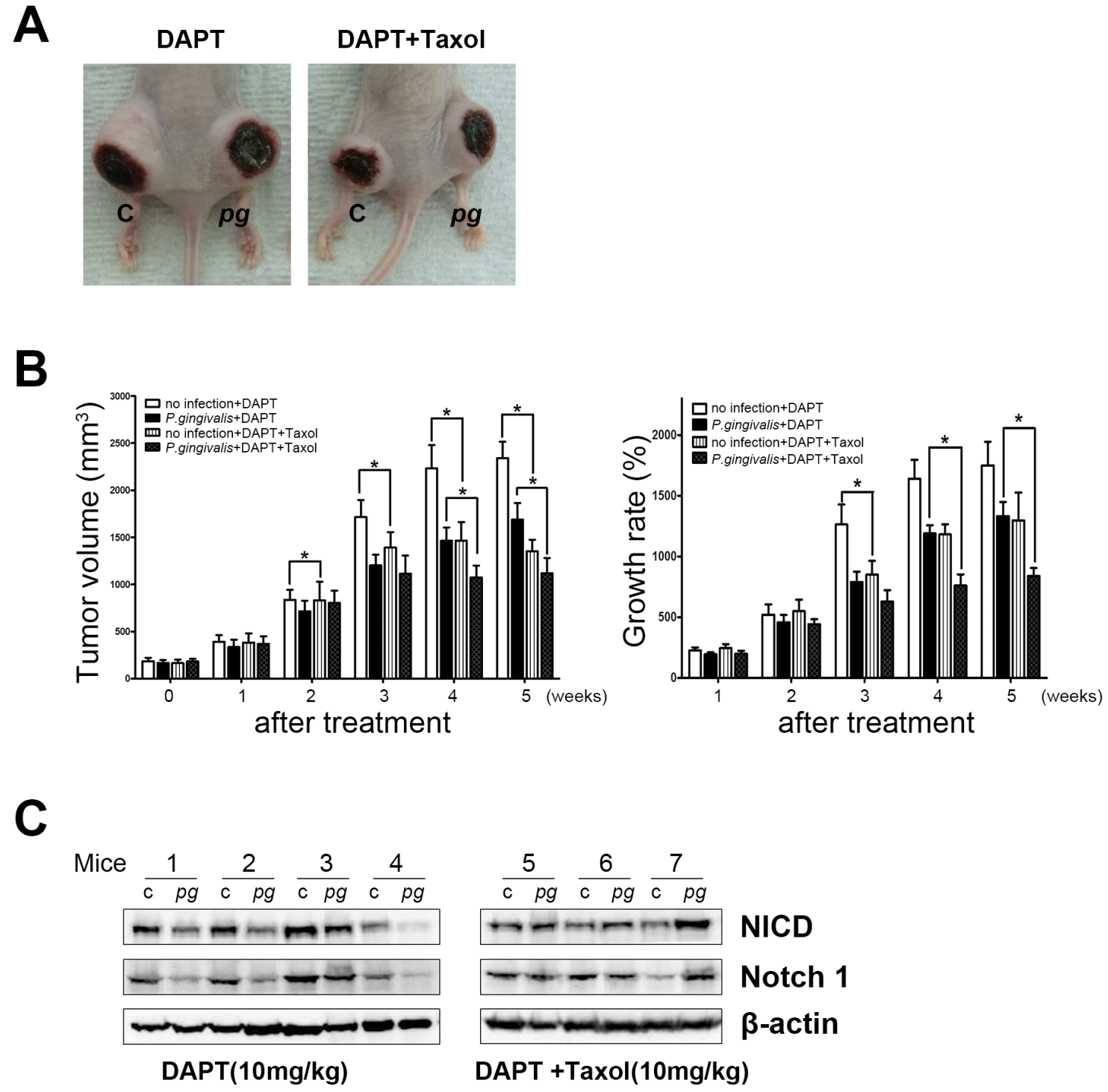

Figure 4. Notch inhibition reduces the chemoresistance of a $P$. gingivalis-infected OSCC xenograft. Mice were treated with $10 \mathrm{mg} / \mathrm{kg}$ of DAPT with or without $5 \mathrm{nM}$ of Taxol twice a week. (A) A representative photograph of mice treated with DAPT or DAPT-Taxol combination chemotherapy was obtained. (B) Tumor volume was measured once a week for 5 weeks. In addition, growth rates of tumor masses were compared and statistically analyzed. Data are presented as mean \pm standard deviation. Significance was assessed using a paired Student's $t$ test. ${ }^{*}, P<0.05$. (C) A Western blot analysis indicated that DAPT treatment decreased NICD expression, and DAPT with Taxol induced no specific changes in NICD levels. 
gingivalis-infected OSCC cells were compared in mice. Briefly, BALB/c mice were administered injections of OSC-20 cells $\left(2 \times 10^{6}\right)$ into their tail vein and sacrificed 30 days later for removal of major organs including the lungs, liver, and kidneys to evaluate distant metastasis of OSC-20 cells. When metastatic lesions were detected, they were localized exclusively to the lung surface, sparing the liver and kidneys. The size of metastatic foci was variable, ranging from microscopic lesions that were not readily detected in gross specimens (Figure 5B, middle panels) to large nodules that replaced large portions of the lung lobes (Figure 5B, right panels). Indeed, the repetitive exposure of OSC-20 cells to $P$. gingivalis for 5 weeks resulted in metastatic foci in the lung tissues of all mice tested (six out of six). Importantly, four of six mice exhibited the formation of macronodules. In contrast, only one mouse injected with uninfected OSC-20 cells developed macronodules in the lungs. As a result, a fewer number of metastatic foci had formed in mice injected with uninfected OSC-20 cells than in mice injected with
A

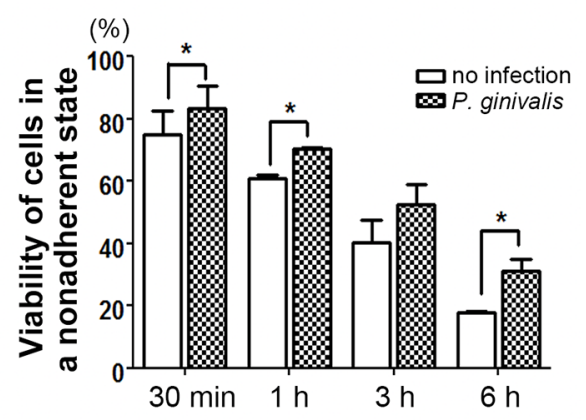

C
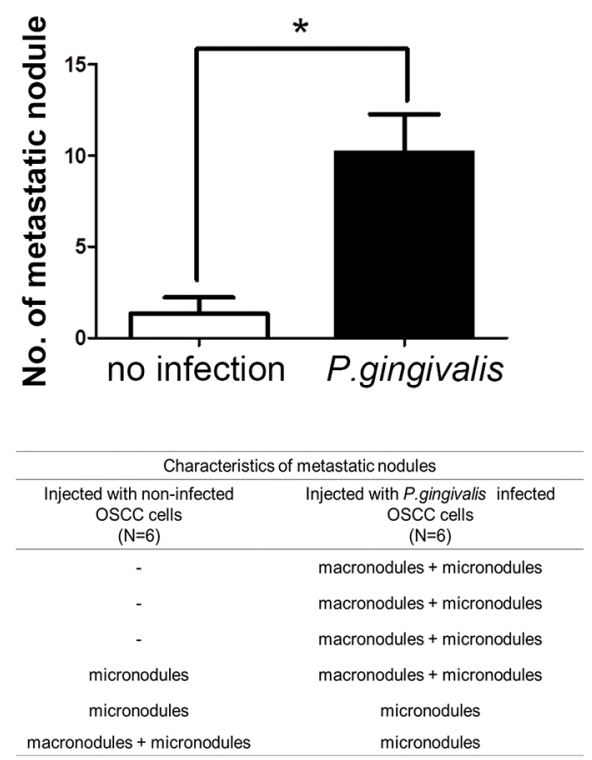

B

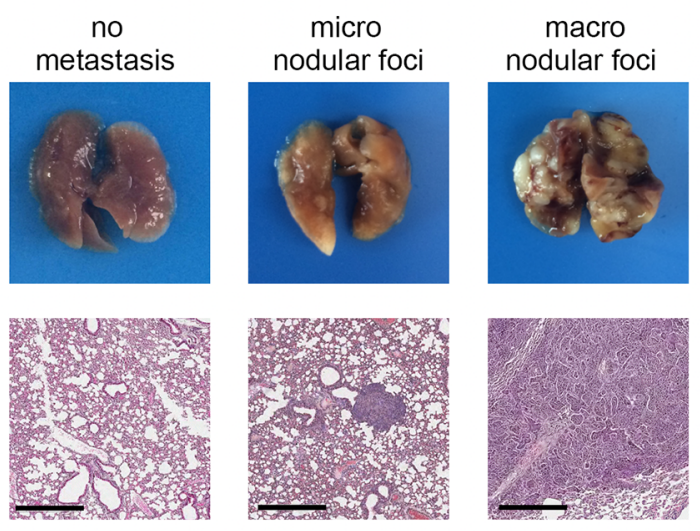

D
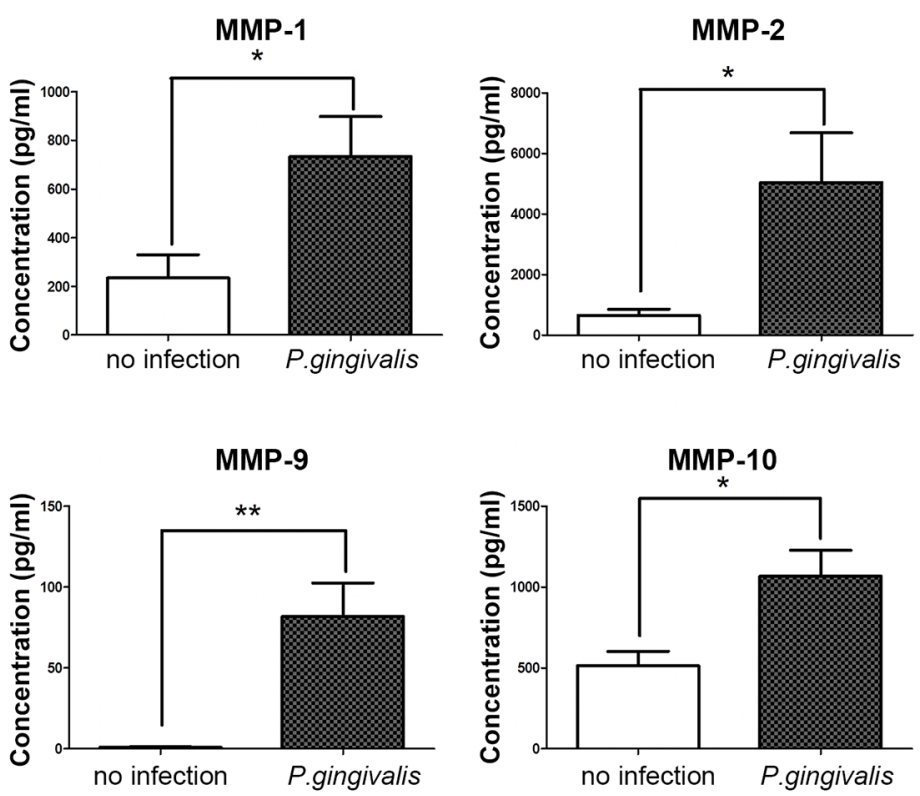

Figure 5. Sustained infection with $P$. gingivalis increased the pulmonary metastasis of OSCC in vivo. (A) Viability of $P$. gingivalis-infected OSCC cells in nonadherent and agitated states was compared with uninfected cells. (B) Representative photographs of pulmonary metastatic foci were obtained at 30 days after intravenous injection of OSC-20 cells into the tail vein. Images of $\mathrm{H} \& \mathrm{E}$ sections were taken to display micronodular and macronodular metastatic lesions in lung tissues. Metastatic nodules which were grossly identified were considered macrodular foci. The nodules which were only validated under light microscopy were counted as micronodular foci, and the sizes of the micronodules were generally no more than $500 \mu \mathrm{m}$ in diameter. Scale bars, $500 \mu \mathrm{m}$. (C) The number of metastatic nodules in lung tissue were counted and analyzed. In addition, characteristics of metastatic lung nodules from mice were investigated and compared depending on injected cell types. Data are presented as mean \pm standard deviation. Significance was assessed using a paired or unpaired Student's $t$ test. * $P<0.05$; **, $P<0.01$. (D) Serum levels of human MMPs in mice injected with uninfected or $P$. gingivalis-infected OSC-20 cells were analyzed using a Luminex assay. 
P. gingivalis-infected cells (Figure 5C). In addition to metastatic foci, the mice injected with $P$. gingivalis infected OSC-20 cells showed higher serum levels of human MMPs including MMP-1, MMP-2, MMP-9, and MMP-10, in contrast to low to nearly undetectable MMPs in mice injected with uninfected OSC-20 cells (Figure 5D). Such low MMP levels may reflect the limited invasive ability of uninfected OSC-20 cells or alternatively reflect a reduced amount of MMPs released from only a few microscopic metastatic lesions.

\section{DISCUSSION}

While acceleration of tumor growth by inflammation-driven mechanisms is well established [21], our understanding of tumor cell responses to microbial infection-induced inflammation can be complicated by multiple factors, including the types of host tumor cells and status of bacterial cells and/or host tumor cells. For example, previous studies have reported that live and heatkilled $P$. gingivalis infections promoted host cell survival and cellular apoptosis, respectively [22-24]. In other studies, live $P$. gingivalis induced apoptosis in trophoblasts but enhanced proliferation in gingival epithelial cells by modulating cell cycle progression $[25,26]$. Considering these complex results, no clear conclusion has been drawn at this moment on the response of tumor cells to $P$. gingivalis infection.

There have been only a few in vivo studies that investigated the effect of $P$. gingivalis on tumor growth. According to one of these studies, growth of $\mathrm{C} 26$ colon carcinoma was accelerated by a $P$. gingivalis GroEL treatment in $\mathrm{BALB} / \mathrm{c}$ mice [27]. Our previous in vitro analysis indicated that a transient $P$. gingivalis infection suppressed host cell proliferation in Ca9-22 OSCC cells by inducing G1 arrest and autophagy [19]. Furthermore, we have reported the reduced proliferation of Ca9-22 OSCC cells resulting from a prolonged and repeated infection with $P$. gingivalis that mimicked chronic inflammation [15]. A similar suppressive effect was also found with $P$. gingivalis in this study, as evidenced by reduced tumor growth in BALB/c xenograft mice harboring OSC20 OSCC cells that were chronically infected with $P$. gingivalis. It is possible that soluble factors released from $P$. gingivalis may promote cell proliferation and contribute to tumor growth, but $P$. gingivalis itself may suppress proliferation of oral cancer cells and tumor mass growth, potentially by cell cycle arrest and autophagy, as we reported, or by residual immune reaction to signals from $P$. gingivalis in $\mathrm{BALB} / \mathrm{c}$ mice.

In addition to its effect on tumor growth, our previous report demonstrated that prolonged and repeated infection with $P$. gingivalis induced epithelial mesenchymal transition and resistance to Taxol and slowed proliferation cycles in OSCC cells. The cells also displayed higher expression levels of CD44 and CD133, representative indicators for cancer stemness [15]. A number of signaling cascades, including the Notch1 pathway, have been recently linked to formation of CSCs, development of chemoresistance, and acquisition of an epithelial-mesenchymal transition (EMT) phenotype [28]. Our findings of enhanced NICD signaling in OSCC cells induced by chronic $P$. gingivalis infection (Figure 2), as well as decreased sensitivity of NICD-overexpressing OSCC cells to Taxol (Figure 3), suggest that chronic $P$. gingivalis infection plays an important role in allowing OSCC cells to develop chemoresistance in a Notch1dependent manner. In addition, enhanced sensitivity of $P$. gingivalis-infected tumor xenografts to combinations of Taxol and DAPT, an inhibitor of Notch activation, further supports that the Notch signaling pathway has a central role in mediating the chemoresistance of chronically infected oral cancer cells.

Our results are in line with other studies that demonstrated the critical role of the Notch signaling pathway in the regulation of CSCs and chemoresistance $[17,18,29]$. Recent reports have also demonstrated the enhanced efficacy of chemotherapeutic drugs including docetaxel and paclitaxel when combined with DAPT in prostate cancer xenograft models and all platinumresistant ovarian tumors, respectively [30-32]. While these findings suggest the need of combined Notch inhibition to improve efficacy of various chemotherapeutic reagents, whether a similar approach can be directly applied to target chronically inflamed cancer, especially periodontitisassociated oral cancer has never been examined. To our knowledge, our current results from both in vitro and in vivo analyses present the first experimental evidence supporting the usefulness of Notch inhibition in concert with conventional cytotoxic chemotherapy to effectively cure chronically inflamed OSCC in the presence of periodontitis-causing pathogens.

Experimental evidence from mouse cancer models and human cancer studies has demonstrated the tight association of chronic inflammation with tumorigenesis and tumor progression. However, our current understanding regarding inflammation in a metastatic cascade is mainly restricted to the effects of inflammatory mediators on metastasis, including TNF- $\alpha$, IL- 1 beta, and TGF-beta, as well as relevant molecular mechanisms [33]. A limited number of experimental studies have demonstrated the effect of bacterial infection on cancer metastasis with metastatic tumor growth promoted by the administration of bacterial LPS in mice [34-36]. It remains unknown whether bacterial pathogens such as $P$. gingivalis can directly influence the metastatic progression of oral cancer. A recent report indicated that a chronic infection involving $P$. gingivalis/Fusobacterium nucleatum through oral administration markedly enhanced the invasiveness of chemically induced OSCC in mice [37]. Herein, we presented the first experimental and molecular evidence demonstrating that OSCC combined with repetitive 
P. gingivalis infections resembling chronic periodontitis developed higher metastatic potential through the activation of the Notch signaling pathway, a key molecular player modulating the EMT and tumor angiogenesis. Thus, our findings may provide a rationale for a combined therapeutic approach in patients with oral cancer consisting of anti-inflammatory regimens and periodontitis treatments along with chemotherapy to decrease the prometastatic activity of chronically inflamed cancer cells.

In summary, we presented in vitro and in vivo experimental evidence supporting the idea that sustained infection with $P$. gingivalis, a major pathogen responsible for chronic periodontitis, promotes distant metastasis of oral cancer, as well as its resistance to anti-cancer agents. Our results suggest that resolution of chronic periodontitis may serve as a promising therapeutic target for metastatic and chemoresistant oral cancers.

\section{MATERIALS AND METHODS}

\section{Cancer cell and bacterial cultures}

The human OSCC cell line, OSC-20, was cultured in a 1:1 mixture of Dulbecco's Modified Eagle's medium and Ham's F-12 nutrient mixture (DME/F12; Hyclone, Logan, UT), supplemented with $10 \%$ fetal bovine serum (FBS; Atlas Biologicals, Fort Collins, CO), streptomycin $(100 \mu \mathrm{g} / \mathrm{mL})$, and amoxicillin and penicillin $(100 \mathrm{IU} / \mathrm{mL})$ (Gibco-Invitrogen Corporation, Rockville, MD) at $37^{\circ} \mathrm{C}$ in a $5 \% \mathrm{CO}_{2}$ incubator. The $P$. gingivalis strain, 381 , was cultured anaerobically and grown in a GAM broth (Nissui, Tokyo, Japan) at $37^{\circ} \mathrm{C}$.

\section{Infection of OSCC cells with $P$. gingivalis}

The OSCC cells were infected with a live $P$. gingivalis strain 381 at an MOI (multiplicity of infection) of $1: 100$ for $3 \mathrm{~h}$ at $37^{\circ} \mathrm{C}$ in $5 \% \mathrm{CO}_{2}$. Then, the cells were washed with PBS and cultured in fresh media until the next infection or harvest. Controls were subjected to the same media changes and wash conditions, without bacterial inoculation.

\section{Western blot analysis}

Western blot analysis was performed according to our previous procedures $[15,19]$. Briefly, approximately $40 \mu \mathrm{g}$ of protein from each sample was denatured and then loaded in each lane of $10-12 \%$ PAGE gel. Subsequently, proteins were transferred onto a PDVF membrane and blocked for $1 \mathrm{~h}$, incubated with primary antibodies overnight, and finally incubated with a horseradish peroxidase-conjugated secondary antibody (Santa Cruz Biotechnology, Santa Cruz, CA). The following primary antibodies were used: Notch1, Notch intracellular domain, and actin.

\section{RNA extraction and real-time PCR}

Total RNA was extracted from cells using an RNeasy Mini kit (Qiagen, Hilden, Germany) according to the manufacturer's instructions. cDNA was synthesized, and bacterial 16S rRNA, along with the mRNAs of Hes 1 and Hey and GAPDH, was detected using 2-step quantitative real-time PCR with a QuantiTect reverse transcription kit (Qiagen) and TOPreal SYBR Green PCR Kit (Enzynomics, Seoul, South Korea) in an ABI 7500 real-time PCR detection system (Applied Biosystems, Foster City, CA).

\section{Cell transfection}

Subconfluent OSC-20 cells were seeded in 6-cm plates and transfected on the following day with $2 \mu \mathrm{g}$ of plasmid DNA using a FuGENE 6 reagent (Roche Diagnostic Corp., Penzberg, Germany), according to the manufacturer's recommendations. The amount of transfected DNA was kept constant with the addition of appropriate amounts of the parental empty vectors.

\section{Luciferase assay}

OSC-20 cells were seeded into 24-well plates, and $4 \times \mathrm{CSL}$ reporter plasmids $(0.5 \mu \mathrm{g}$ each) were separately transfected into the cells. After $48 \mathrm{~h}$, the cells were lysed in a passive lysis buffer; luciferase activity was measured with a Dual-Luciferase Reporter Assay System (Promega, Madison, WI); and target promoter-driven firefly luciferase activity was normalized to that of the Renilla control. Each experiment was repeated three times.

\section{MTT assay}

The cells were treated for $24 \mathrm{~h}$ or $48 \mathrm{~h}$ with various concentrations of Taxol $24 \mathrm{~h}$ after transfection. Subsequently, the medium was removed, and the cells were incubated in an MTT solution $(1 \mathrm{mg} / \mathrm{mL} ; 100 \mu \mathrm{L} /$ well) at $37^{\circ} \mathrm{C}$ for $4 \mathrm{~h}$. The supernatant was then removed, and $100 \mu \mathrm{L}$ of dimethyl sulfoxide (DMSO) was added to each well to dissolve the insoluble formazan. The 96-well plates were shaken for $10 \mathrm{~min}$, and absorbance at 545 $\mathrm{nm}$ was measured using a microplate reader. Then, the percentage of cell growth was calculated based on $100 \%$ growth without treatment with Taxol.

\section{Cell viability in a nonadherent and agitated state}

The cells were harvested and placed into a 15$\mathrm{mL}$ polystyrene tube in the presence of cell media. The tubes were continuously shaken at $37{ }^{\circ} \mathrm{C}$ using a shaking incubator $(200 \mathrm{rpm} / \mathrm{min})$; then, the number of viable and nonviable cells was counted at the indicated time points. 


\section{Luminex assay}

Levels of matrix metalloproteinases (MMPs) in the serum of mice were measured using a Milliplex Map Human MMP panel 2 kit (R \& D system, Minneapolis, MN). The Luminex 200 platform, coupled with BioRad Bio-Plex software (BioRad), was used to measure the MMP levels according to the manufacturer's protocols.

\section{Nude mouse xenograft chemotherapeutic experiment}

Six-week-old BALB/c male mice (18-20 g) were housed in a specific pathogen-free environment at the Experimental Animal Center of Pusan National University in pressurized, ventilated cages according to institutional regulations. For a nude mouse xenograft chemotherapeutic experiment, each $2 \times 10^{6}$ of uninfected and $P$. gingivalis infected OSC-20 cells were injected subcutaneously into the left and right back, respectively, of nude mice. Two weeks after injection, the tumor was visible, and then, Taxol treatment was initiated. Taxol, purchased from Sigma-Aldrich (St. Louis, MO), was initially dissolved in $100 \%$ DMSO at a concentration of $10 \mathrm{mg} / \mathrm{mL}$ and stored at $-20{ }^{\circ} \mathrm{C}$. PBS was used as a negative control vehicle. For chemotherapy experiments, $10 \mathrm{mg} / \mathrm{kg}$ of Taxol or vehicle was infused intraperitoneally twice a week for 4 weeks. All animal practices in this study were approved and conducted in accordance with the guidelines of the Association of Laboratory Animal Science of Pusan National University (PNU-2015-0893).

\section{Histology}

Tumor mass and lung tissue samples were fixed in $10 \%$ neutral-buffered formalin solution and embedded in paraffin. Tissue sections were stained with hematoxylin and eosin ( $\mathrm{H} \& \mathrm{E})$ to examine tissue morphology, and $\mathrm{H} \&$ E stained sections were observed under light microscopy.

\section{OSCC metastasis experiment}

We injected $2 \times 10^{6}$ OSC-20 cells diluted in 100 $\mu \mathrm{L}$ of PBS into the tail vein of 7 -week-old BALB/c male mice after they were housed for 1 week. On the day of the injection, the mice were divided randomly into two groups. The mice were sacrificed after 30 days using Zoletil/Rompun intravenous injection, and the lungs were removed and fixed in $10 \%$ formalin. For further analysis, the lung tissue samples were trimmed, processed, embedded, sectioned, and stained with $\mathrm{H}$ \& E to examine tissue morphology. All mice were fed $a d$ libitum and housed in accordance with the guidelines of the Association of Laboratory Animal Science of Pusan National University (PNU-2015-0893).

\section{Statistical analysis}

Statistical analysis was performed with GraphPad Prism 5.03 (GraphPad Software, Inc., La Jolla, CA). Data were analyzed using a Student's $t$ test to compare control and $P$. gingivalis - infected groups. All data are presented as mean \pm SD. $P$ values less than 0.05 were considered significant.

\section{Author contributions}

BH Woo and JM Song conceived and participated in this project, and $\mathrm{BH}$ Woo and DJ Kim performed these experiments. JI Choi, SJ Kim, BS Park are involved in study design. JI Choi, SJ Kim, and HR Park made contribution to data analysis and interpretation. JH Lee made contribution to statistical analysis. BH Woo, JM Song, JH Lee and HR Park wrote the manuscript. All of the authors have reviewed the final paper.

\section{CONFLICTS OF INTEREST}

The authors declare no conflicts of interest.

\section{FUNDING}

This work was supported by Basic Science Research Program through National Research Foundation of Korea (NRF) funded by the Ministry of Education (2014R1A1A2A16050554).

\section{REFERENCES}

1. Deng H, Sambrook PJ, Logan RM. The treatment of oral cancer: an overview for dental professionals. Australian Dental Journal. 2011; 56: 244-52, 341.

2. Zhang L, Song X, Mohri Y, Qiao L. Role of inflammation and tumor microenvironment in the development of gastrointestinal cancers: what induced pluripotent stem cells can do?. Current stem cell research \& therapy. 2015; 10: 245-250.

3. Rimessi A, Patergnani S, Ioannidi E, Pinton P. Chemoresistance and Cancer-Related Inflammation: Two Hallmarks of Cancer Connected by an Atypical Link, PKCzeta. Frontiers in oncology. 2013; 3: 232.

4. Liu J, Lin PC, Zhou BP. Inflammation fuels tumor progress and metastasis. Current pharmaceutical design. 2015; 21 : 3032-3040.

5. Li Y, Wang L, Pappan L, Galliher-Beckley A, Shi J. IL-1beta promotes stemness and invasiveness of colon cancer cells through Zeb1 activation. Molecular cancer. 2012; 11: 87-4598-11-87. 
6. Bharti R, Dey G, Mandal M. Cancer development, chemoresistance, epithelial to mesenchymal transition and stem cells: A snapshot of IL-6 mediated involvement. Cancer letters. 2016; 375: 51-61.

7. Wang Q, Zhou X, Huang D. Role for Porphyromonas gingivalis in the progression of atherosclerosis. Medical hypotheses. 2009; 72: 71-73.

8. MacDonagh L, Gray SG, Breen E, Cuffe S, Finn SP, O'Byrne KJ, Barr MP. Lung cancer stem cells: The root of resistance. Cancer letters. 2016; 372: 147-156.

9. Fitzpatrick SG, Katz J. The association between periodontal disease and cancer: a review of the literature. Journal of dentistry. 2010; 38: 83-95.

10. Meyer MS, Joshipura K, Giovannucci E, Michaud DS. A review of the relationship between tooth loss, periodontal disease, and cancer. Cancer causes \& control. 2008; 19: 895-907.

11. Tezal M, Sullivan MA, Hyland A, Marshall JR, Stoler D, Reid ME, Loree TR, Rigual NR, Merzianu M, Hauck L, Lillis C, Wactawski-Wende J, Scannapieco FA. Chronic periodontitis and the incidence of head and neck squamous cell carcinoma. Cancer epidemiology, biomarkers \& prevention. 2009; 18: 2406-2412.

12. Laprise C, Shahul HP, Madathil SA, Thekkepurakkal AS, Castonguay G, Varghese I, Shiraz S, Allison P, Schlecht NF, Rousseau MC, Franco EL, Nicolau B. Periodontal diseases and risk of oral cancer in Southern India: Results from the HeNCe Life study. International journal of cancer. 2016; 139: 1512-1519.

13. Inaba $\mathrm{H}$, Sugita $\mathrm{H}$, Kuboniwa $\mathrm{M}$, Iwai $\mathrm{S}$, Hamada $\mathrm{M}$, Noda T, Morisaki I, Lamont RJ, Amano A. Porphyromonas gingivalis promotes invasion of oral squamous cell carcinoma through induction of proMMP9 and its activation. Cellular microbiology. 2014; 16: 131-145.

14. Ha NH, Park DG, Woo BH, Kim da J, Choi JI, Park BS, Kim YD, Lee JH, Park HR. Porphyromonas gingivalis increases the invasiveness of oral cancer cells by upregulating IL-8 and MMPs. Cytokine. 2016; 86: 64-72.

15. Ha NH, Woo BH, Kim DJ, Ha ES, Choi JI, Kim SJ, Park BS, Lee JH, Park HR. Prolonged and repetitive exposure to Porphyromonas gingivalis increases aggressiveness of oral cancer cells by promoting acquisition of cancer stem cell properties. Tumour biology. 2015; 36: 9947-9960.

16. Bin Hafeez B, Adhami VM, Asim M, Siddiqui IA, Bhat KM, Zhong W, Saleem M, Din M, Setaluri V, Mukhtar H. Targeted knockdown of Notch1 inhibits invasion of human prostate cancer cells concomitant with inhibition of matrix metalloproteinase- 9 and urokinase plasminogen activator. Clinical cancer research. 2009; 15: 452-459.

17. Domingo-Domenech J, Vidal SJ, Rodriguez-Bravo V, Castillo-Martin M, Quinn SA, Rodriguez-Barrueco R, Bonal DM, Charytonowicz E, Gladoun N, de la IglesiaVicente J, Petrylak DP, Benson MC, Silva JM, et al.
Suppression of acquired docetaxel resistance in prostate cancer through depletion of notch- and hedgehog-dependent tumor-initiating cells. Cancer cell. 2012; 22: 373-388.

18. Patrawala L, Calhoun T, Schneider-Broussard R, Zhou J, Claypool K, Tang DG. Side population is enriched in tumorigenic, stem-like cancer cells, whereas ABCG2+ and ABCG2- cancer cells are similarly tumorigenic. Cancer research. 2005; 65: 6207-6219.

19. Cho TJ, Wee SW, Woo VH, Choi JI, Kim SJ, Shin HI, Lee JH, Park HR. Porphyromonas gingivalis-induced autophagy suppresses cell proliferation through G1 arrest in oral cancer cells. Archives of Oral Biology. 2014; 59: 370-378.

20. Abel EV, Kim EJ, Wu J, Hynes M, Bednar F, Proctor E, Wang L, Dziubinski ML, Simeone DM. The Notch pathway is important in maintaining the cancer stem cell population in pancreatic cancer. PloS one. 2014; 9: e91983.

21. Grivennikov SI, Greten FR, Karin M. Immunity, inflammation, and cancer. Cell. 2010; 140: 883-899.

22. Nakhjiri SF, Park Y, Yilmaz O, Chung WO, Watanabe K, El-Sabaeny A, Park K, Lamont RJ. Inhibition of epithelial cell apoptosis by Porphyromonas gingivalis. FEMS microbiology letters. 2001; 200: 145-149.

23. Yao L, Jermanus C, Barbetta B, Choi C, Verbeke P, Ojcius DM, Yilmaz O. Porphyromonas gingivalis infection sequesters pro-apoptotic Bad through Akt in primary gingival epithelial cells. Molecular oral microbiology. 2010; 25: 89-101.

24. Yilmaz O, Jungas T, Verbeke P, Ojcius DM. Activation of the phosphatidylinositol 3-kinase/Akt pathway contributes to survival of primary epithelial cells infected with the periodontal pathogen Porphyromonas gingivalis. Infection and immunity. 2004; 72: 3743-3751.

25. Inaba H, Kuboniwa M, Bainbridge B, Yilmaz O, Katz J, Shiverick KT, Amano A, Lamont RJ. Porphyromonas gingivalis invades human trophoblasts and inhibits proliferation by inducing G1 arrest and apoptosis. Cellular microbiology. 2009; 11: 1517-1532.

26. Kuboniwa M, Hasegawa Y, Mao S, Shizukuishi S, Amano A, Lamont RJ, Yilmaz O. P. gingivalis accelerates gingival epithelial cell progression through the cell cycle. Microbes and infection / Institut Pasteur. 2008; 10: 122-128.

27. Lin FY, Huang CY, Lu HY, Shih CM, Tsao NW, Shyue SK, Lin CY, Chang YJ, Tsai CS, Lin YW, Lin SJ. The GroEL protein of Porphyromonas gingivalis accelerates tumor growth by enhancing endothelial progenitor cell function and neovascularization. Molecular oral microbiology. 2015; 30: 198-216.

28. Wang Z, Li Y, Ahmad A, Azmi AS, Banerjee S, Kong D, Sarkar FH. Targeting Notch signaling pathway to overcome drug resistance for cancer therapy. Biochimica et biophysica acta. 2010; 1806: 258-267.

29. McAuliffe SM, Morgan SL, Wyant GA, Tran LT, Muto KW, Chen YS, Chin KT, Partridge JC, Poole BB, Cheng KH, 
Daggett J Jr, Cullen K, Kantoff E, et al. Targeting Notch, a key pathway for ovarian cancer stem cells, sensitizes tumors to platinum therapy. Proceedings of the National Academy of Sciences of the United States of America. 2012; 109: E2939-48.

30. Cui D, Dai J, Keller JM, Mizokami A, Xia S, Keller ET. Notch Pathway Inhibition Using PF-03084014, a gammaSecretase Inhibitor (GSI), Enhances the Antitumor Effect of Docetaxel in Prostate Cancer. Clinical cancer research. 2015; 21: 4619-4629.

31. Groeneweg JW, DiGloria CM, Yuan J, Richardson WS, Growdon WB, Sathyanarayanan S, Foster R, Rueda BR. Inhibition of notch signaling in combination with Paclitaxel reduces platinum-resistant ovarian tumor growth. Frontiers in oncology. 2014; 4: 171.

32. Wang M, Ma X, Wang J, Wang L, Wang Y. Pretreatment with the gamma-secretase inhibitor DAPT sensitizes drugresistant ovarian cancer cells to cisplatin by downregulation of Notch signaling. International journal of oncology. 2014; 44: 1401-1409.

33. Kim S, Karin M. Role of TLR2-dependent inflammation in metastatic progression. Annals of the New York Academy of Sciences. 2011; 1217: 191-206.
34. Pidgeon GP, Harmey JH, Kay E, Da Costa M, Redmond HP, Bouchier-Hayes DJ. The role of endotoxin/ lipopolysaccharide in surgically induced tumour growth in a murine model of metastatic disease. British journal of cancer. 1999; 81: 1311-1317.

35. Luo JL, Maeda S, Hsu LC, Yagita H, Karin M. Inhibition of NF-kappaB in cancer cells converts inflammation- induced tumor growth mediated by TNFalpha to TRAIL-mediated tumor regression. Cancer cell. 2004; 6: 297-305.

36. Harmey JH, Bucana CD, Lu W, Byrne AM, McDonnell S, Lynch C, Bouchier-Hayes D, Dong Z. Lipopolysaccharideinduced metastatic growth is associated with increased angiogenesis, vascular permeability and tumor cell invasion. International journal of cancer. 2002; 101: 415-422.

37. Binder Gallimidi A, Fischman S, Revach B, Bulvik R, Maliutina A, Rubinstein AM, Nussbaum G, Elkin M. Periodontal pathogens Porphyromonas gingivalis and Fusobacterium nucleatum promote tumor progression in an oral-specific chemical carcinogenesis model. Oncotarget. 2015; 6: 22613-22623. doi: 10.18632/oncotarget.4209. 